\title{
Front Matter: Volume 8502
}

, "Front Matter: Volume 8502," Proc. SPIE 8502, Advances in X-Ray/EUV Optics and Components VII, 850201 (13 November 2012); doi: $10.1117 / 12.2012084$

EDIE Event: SPIE Optical Engineering + Applications, 2012, San Diego, California, SPIE. United States 


\title{
PROCEEDINGS OF SPIE
}

\section{Advances in X-Ray/EUV Optics and Components VII}

\author{
Shunji Goto \\ Christian Morawe \\ Ali M. Khounsary \\ Editors
}

13 August 2012

San Diego, California, United States

Sponsored and Published by

SPIE 
The papers included in this volume were part of the technical conference cited on the cover and title page. Papers were selected and subject to review by the editors and conference program committee. Some conference presentations may not be available for publication. The papers published in these proceedings reflect the work and thoughts of the authors and are published herein as submitted. The publisher is not responsible for the validity of the information or for any outcomes resulting from reliance thereon.

Please use the following format to cite material from this book:

Author(s), "Title of Paper," in Advances in X-Ray/EUV Optics and Components VII, edited by Shunji Goto, Christian Morawe, Ali M. Khounsary, Proceedings of SPIE Vol. 8502 (SPIE, Bellingham, WA, 2012) Article CID Number.

ISSN: 0277-786X

ISBN: 9780819492197

Published by

SPIE

P.O. Box 10, Bellingham, Washington 98227-0010 USA

Telephone +1 3606763290 (Pacific Time) · Fax +1 3606471445

SPIE.org

Copyright (@ 2012, Society of Photo-Optical Instrumentation Engineers.

Copying of material in this book for internal or personal use, or for the internal or personal use of specific clients, beyond the fair use provisions granted by the U.S. Copyright Law is authorized by SPIE subject to payment of copying fees. The Transactional Reporting Service base fee for this volume is $\$ 18.00$ per article (or portion thereof), which should be paid directly to the Copyright Clearance Center (CCC), 222 Rosewood Drive, Danvers, MA 01923. Payment may also be made electronically through CCC Online at copyright.com. Other copying for republication, resale, advertising or promotion, or any form of systematic or multiple reproduction of any material in this book is prohibited except with permission in writing from the publisher. The CCC fee code is 0277-786X/12/\$18.00.

Printed in the United States of America.

Publication of record for individual papers is online in the SPIE Digital Library.

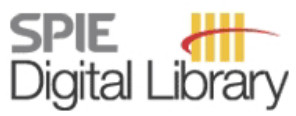

SPIEDigitalLibrary.org

Paper Numbering: Proceedings of SPIE follow an e-First publication model, with papers published first online and then in print and on CD-ROM. Papers are published as they are submitted and meet publication criteria. A unique, consistent, permanent citation identifier (CID) number is assigned to each article at the time of the first publication. Utilization of CIDs allows articles to be fully citable as soon as they are published online, and connects the same identifier to all online, print, and electronic versions of the publication. SPIE uses a six-digit CID article numbering system in which:

- The first four digits correspond to the SPIE volume number.

- The last two digits indicate publication order within the volume using a Base 36 numbering

system employing both numerals and letters. These two-number sets start with 00, 01, 02, 03, 04, $05,06,07,08,09,0 A, 0 B \ldots 0 Z$, followed by 10-1Z, 20-2Z, etc.

The CID Number appears on each page of the manuscript. The complete citation is used on the first page, and an abbreviated version on subsequent pages. Numbers in the index correspond to the last two digits of the six-digit CID Number. 


\section{Contents}

vii Conference Committee

SESSION 1 MULTILAYER OPTICS

850202 Advanced multilayer Lave lens fabrication at NSLS-II [8502-1]

R. Conley, N. Bouet, J. Zhou, H. Yan, Y. Chu, K. Laver, J. Miller, Brookhaven National Lab. (United States); L. Chu, Northport High School (United States); N. Jahedi, Argonne National Lab. (United States)

850204 Aperiodic multilayer mirrors for attosecond soft $\mathbf{x}$-ray pulses [8502-3]

A. Guggenmos, M. Hofstetter, Ludwig-Maximilians-Univ München (Germany) and Max-Planck-Institut für Quantenoptik (Germany); R. Rauhut, B. Nickel, Ludwig-MaximiliansUniv München (Germany); E. Gullikson, Lawrence Berkeley National Lab. (United States); U. Kleineberg, Ludwig-Maximilians-Univ. München (Germany) and Max-Planck-Institut für Quantenoptik (Germany)

850206 A soft $x$-ray beam-splitting multilayer optic for the NASA GEMS Bragg Reflection Polarimeter [8502-25]

R. Allured, The Univ. of lowa (United States); M. Fernández-Perea, R. Soufli, J. B. Alameda, Lawrence Livermore National Lab. (United States); A. Maxwell, The Univ. of lowa (United States); M. J. Pivovaroff, Lawrence Livermore National Lab. (United States); E. M. Gullikson, Lawrence Berkeley National Lab. (United States); P. Kaaret, The Univ. of lowa (United States)

\section{SESSION 2 SIMULATIONS AND CODES}

850208 Application of wave optical simulations to focusing $x$-ray multilayers [8502-7]

M. Osterhoff, European Synchrotron Radiation Facility (France) and Georg-August-Univ. Göttingen (Germany); C. Morawe, European Synchrotron Radiation Facility (France)

850209 Coded aperture imaging for fluorescent $x$-rays [8502-28]

A. Haboub, A. A. MacDowell, S. Marchesini, D. Y. Parkinson, Lawrence Berkeley National Lab. (United States)

\section{SESSION 3 MIRRORS AND MONOCHROMATORS}

8502 OA Diamond double-crystal monochromator at SPring-8 [8502-9]

S. Goto, H. Yamazaki, Y. Shimizu, M. Suzuki, N. Kawamura, M. Mizumaki, Japan Synchrotron Radiation Research Institute (Japan); M. Yabashi, K. Tamasaku, T. Ishikawa, RIKEN (Japan)

$8502 \mathrm{OB} \quad$ Parameterizing the deformation of $\mathrm{LN}_{2}$-cooled silicon monochromator crystals with modified linear power density funtion [8502-10]

R. Huang, D. H. Bilderback, Cornell Univ. (United States) 
$85020 \mathrm{C}$ On the limitations of liquid nitrogen cooling of high heat load $\mathrm{x}$-ray monochromators [8502-11]

A. Khounsary, P. Strons, N. Kujala, A. Macrander, Argonne National Lab. (United States)

8502 OD Novel design of a large $x$-ray optical system for astrophysical application [8502-12]

L. Pina, R. Hudec, V. Tichy, Czech Technical Univ. in Prague (Czech Republic); A. Inneman,

D. Cerna, J. Marsik, V. Marsikova, Rigaku Innovative Technologies Europe (Czech

Republic); W. Cash, A. F. Shipley, B. R. Zeiger, T. D. Rogers, Univ. of Colorado at Boulder

(United States); R. Melich, Institute of Plasma Physics of the ASCR, v.v.i. (Czech Republic)

8502 OE Minimizing the mirror distortion for subarcsecond imaging in the Hi-C EUV telescope [8502-13]

W. A. Podgorski, D. Caldwell, K. McCracken, M. P. Ordway, P. N. Cheimets, K. Korreck,

L. Golub, Harvard-Smithsonian Ctr. for Astrophysics (United States); J. Cirtain, K. Kobayashi, NASA Marshall Space Flight Ctr. (United States)

8502 OF Automated finishing of diamond turned dies for hard $\mathrm{x}$-ray and EUV optics replication [8502-14]

A. T. H. Beaucamp, Chubu Univ. (Japan) and Zeeko Ltd. (United Kingdom); Y. Namba,

Chubu Univ. (Japan); R. R. Freeman, Zeeko Ltd. (United Kingdom)

$85020 \mathrm{G}$ Video-based beam position monitoring at CHESS (Invited Paper) [8502-15]

P. Revesz, A. Pauling, T. Krawczyk, Cornell Univ. (United States); K. J. Kelly, Univ. of Notre

Dame (United States)

$8502 \mathrm{OH} \quad$ Temporal modulation of synchrotron x-rays using torsional MEMS mirrors [8502-16]

D. A. Walko, I. W. Jung, D. Lopez, D. Mukhopadhyay, C. P. Schwartz, G. K. Shenoy, J. Wang, Argonne National Lab. (United States)

8502 Ol Recent tests of $x$-ray spectrometers using polycapillary optics [8502-17]

S. Heald, Argonne National Lab. (United States); G. T. Seidler, D. Mortensen, B. Mattern, Univ. of Washington (United States); J. A. Bradley, Lawrence Livermore National Lab. (United States); N. Hess, M. Bowden, Pacific Northwest National Lab. (United States)

8502 0J Enhanced $\mathbf{x}$-ray angular dispersion and $\mathbf{x}$-ray spectrographs with resolving power beyond $10^{8}$ [8502-18]

Y. Shvyd'ko, Argonne National Lab. (United States)

8502 OK 3D x-ray fluorescence microscopy with $1.7 \mu \mathrm{m}$ resolution using lithographically fabricated micro-channel arrays [8502-19]

A. R. Woll, D. Agyeman-Budu, D. H. Bilderback, D. Dale, A. Y. Kazimirov, Cornell Univ. (United States); M. Pfeifer, Advanced Design Consulting (United States); T. Plautz, T. Szebenyi, G. Untracht, Cornell Univ. (United States)

$8502 \mathrm{OL}$ Addressable flat-panel $\mathrm{x}$-ray sources for medical, security, and industrial applications [8502-20]

G. Travish, Univ. of California, Los Angeles (United States) and Radius Diagnostics Research, Inc. (United States); F. J. Rangel, M. A. Evans, B. Hollister, K. Schmiedehausen, Radius Diagnostics Research, Inc. (United States) 
8502 OM Achieving optimal flatness and surface roughness properties for novel x-ray optic structures formed by dicing saws [8502-21]

M. Wieczorek, R. Khachatryan, Y. Shvyd'ko, Argonne National Lab. (United States); R. H. Smith, Advanced Dicing Technologies Ltd. (United States); K. Iwasaki, Disco Hi-Tec America, Inc. (United States); S. Miller, J. Qian, X. Huang, L. Assoufid, Argonne National Lab. (United States)

850200 Thermo-mechanical properties of silicon, germanium, diamond, beryllium and silicon carbide for high heat load $x$-ray optics applications [8502-23]

A. Hedayat, Univ. of Illinois at Chicago (United States); A. Khounsary, Argonne National Lab. (United States); F. Mashayek, Univ. of Illinois at Chicago (United States)

8502 OP Upgrade of the beamline 10.0.1 at the advanced light source [8502-26]

Z. Ali, Lawrence Berkeley National Lab. (United States) and Optics Labs. (Pakistan);

Y.-D. Chuang, D. Kilcoyne, A. Aguilar, S.-K. Mo, Z. Hussain, Lawrence Berkeley National Lab. (United States)

Author Index 
Proc. of SPIE Vol. $8502850201-6$

Downloaded From: https://www.spiedigitallibrary.org/conference-proceedings-of-spie on 26 Apr 2023 Terms of Use: https://www.spiedigitallibrary.org/terms-of-use 


\section{Conference Committee}

Program Track Chair

Carolyn A. MacDonald, University at Albany (United States)

Conference Chairs

Shunji Goto, Japan Synchrotron Radiation Research Institute (Japan) Christian Morawe, European Synchrotron Radiation Facility (France)

Ali M. Khounsary, Argonne National Laboratory (United States)

\section{Conference Program Committee}

Lucia Alianelli, Diamond Light Source Ltd. (United Kingdom)

Lahsen Assoufid, Argonne National Laboratory (United States)

Stefan Braun, Fraunhofer-Institut für Werkstoff- und Strahltechnik (Germany)

Shih-Lin Chang, National Tsing Hua University (Taiwan)

Raymond Conley Jr., Brookhaven National Laboratory (United States)

Sultan B. Dabagov, Istituto Nazionale di Fisica Nucleare (Italy)

Christian David, Paul Scherrer Institut (Switzerland)

Hans M. Hertz, Royal Institute of Technology (Sweden)

Keiichi Hirano, KEK-High Energy Accelerator Research Organization (Japan)

Werner H. Jark, Sincrotrone Trieste S.C.p.A. (Italy)

Yasushi Kagoshima, University of Hyogo (Japan)

George A. Kyrala, Los Alamos National Laboratory (United States)

Carolyn A. MacDonald, University at Albany (United States)

Howard A. Padmore, Lawrence Berkeley National Laboratory (United States)

Ladislav Pina, Czech Technical University in Prague (Czech Republic)

Yuriy Ya. Platonov, Rigaku Innovative Technologies, Inc. (United States)

Seungyu Rah, Pohang University of Science and Technology (Korea, Republic of)

Peter Revesz, Cornell University (United States)

Wa'el Salah, SESAME (Jordan)

Horst Schulte-Schrepping, Deutsches Elektronen-Synchrotron

(Germany)

Regina Soufli, Lawrence Livermore National Laboratory

(United States)

Stanislav Stoupin, Argonne National Laboratory (United States)

Jörg Wiesmann, Incoatec GmbH (Germany) 
Stephen W. Wilkins, Commonwealth Scientific and Industrial Research Organisation (Australia)

Makina Yabashi, Japan Synchrotron Radiation Research Institute (Japan) and RIKEN (Japan)

Kazuto Yamauchi, Osaka University (Japan)

Brian W. Yates, Canadian Light Source Inc. (Canada)

\section{Session Chairs}

1 Multilayer Optics

Christian Morawe, European Synchrotron Radiation Facility (France)

Regina Soufli, Lawrence Livermore National Laboratory (United States)

2 Simulations and Codes

Shunji Goto, Japan Synchrotron Radiation Research Institute (Japan)

Carolyn A. MacDonald, University at Albany (United States)

3 Mirrors and Monochromators

Kazuto Yamauchi, Osaka University (Japan)

Ladislav Pina, Czech Technical University in Prague (Czech Republic)

4 Beamlines and Instrumentation

Ali Khounsary, Argonne National Laboratory (United States)

Stanislav Stoupin, Argonne National Laboratory (United States) 